PROCEEDINGS OF THE

AMERICAN MATHEMATICAL SOCIETY

Volume 136, Number 7, July 2008, Pages 2425-2428

S 0002-9939(08)08752-2

Article electronically published on March 7, 2008

\title{
HYPONORMAL TOEPLITZ OPERATORS AND ZEROS OF POLYNOMIALS
}

\author{
TAKAHIKO NAKAZI
}

(Communicated by Joseph A. Ball)

\begin{abstract}
The problem of hyponormality for Toeplitz operators with (trigonometric) polynomial symbols is studied. We give a necessary and sufficient condition using the zeros of the analytic polynomial induced by the Fourier coefficients of the symbol.
\end{abstract}

Let $L^{p}$ be the Lebesgue space on the unit circle $T$ and let $H^{p}$ be the corresponding Hardy space for $1 \leq p \leq \infty$. The Toeplitz operator $T_{\phi}$ with symbol $\phi$ in $L^{\infty}$ is the operator on $H^{2}$ defined by $T_{\phi} f=P(\phi f)$ for $f$ in $H^{2}$, where $P$ is the orthogonal projection from $L^{2}$ onto $H^{2}$. In this paper, we are interested in when $T_{\phi}$ is hyponormal.

Two characterizations of the hyponormality of $T_{\phi}$ are known as the following :

(I) Suppose $\phi_{1}$ and $\phi_{2}$ are functions in $H^{2}$ with $\phi=\phi_{1}+\bar{\phi}_{2}$ in $L^{\infty}$. Then $T_{\phi}$ is hyponormal if and only if there exists a constant $c$ and $a$ function $k$ in $H^{\infty}$ with $\|k\|_{\infty} \leq 1$ such that $\phi_{2}=c+T_{\bar{k}} \phi_{1}$.

(II) $T_{\phi}$ is hyponormal if and only if there exist two functions $k$ and $g$ in $H^{\infty}$ such that $\phi=k \bar{\phi}+g$ and $\|k\|_{\infty} \leq 1$.

The characterization (I) is due to Cowen [1. Cowen [1] and Zhu [6] used this characterization. (II) is due to Nakazi-Takahashi [4, Lemma 1]. It is easy to prove (II) if we use (I). Nakazi-Takahashi [4 and Hwang-Lee [3] used this one. HwangLee [3] established an explicit and useful criterion using (II) when the symbol $\phi$ is a trigonometric polynomial. Their criterion involves the zeros of an analytic polynomial induced by the Fourier coefficients of $\phi$. On the other hand, Zhu [6] gave a characterization which is related to the coefficients of the analytic polynomial induced by the Fourier coefficients of $\phi$, using (I) and a theorem of Schur [5]. In this paper, we give a necessary and sufficient condition which is related to the zeros of an analytic polynomial induced by the Fourier coefficient of $\phi$, using (II) and the Carathéodory-Schur interpolation theorem (cf. 3]).

Received by the editors December 2, 2005, and, in revised form, April 12, 2006.

2000 Mathematics Subject Classification. Primary 47B20, 47B35.

Key words and phrases. Trigonometric Toeplitz operators, hyponormal operator, Pick problem.

This research was partially supported by Grant-in-Aid for Scientific Research, Japan Society for the Promotion of Science. 
Theorem 1. Suppose $\phi$ is a trigonometric polynomial such that

$$
\phi=\bar{z}^{\ell} \prod_{j=1}^{t}\left(z-\alpha_{j}\right) \prod_{j=1}^{s}\left(z-\beta_{j}\right)
$$

where $\ell \geq 1,\left|\alpha_{j}\right|<1$ and $\left|\beta_{j}\right| \geq 1$. When $t=0$ or $s=0$, we assume that

$$
\prod_{j=1}^{t}\left(z-\alpha_{j}\right)=1 \quad \text { or } \quad \prod_{j=1}^{s}\left(1-\beta_{j}\right)=1 .
$$

Let

$$
f=\prod_{j=1}^{t} \frac{z-\alpha_{j}}{1-\bar{\alpha}_{j} z} \quad \text { and } \quad h=\prod_{j=1}^{s} \frac{1-\bar{\beta}_{j} z}{z-\beta_{j}} .
$$

Then $T_{\phi}$ is hyponormal if and only if $2 \ell \leq t+s$ and there exists a solution $a_{0}, \ldots, a_{\ell-1}$ of the linear system of equations

$$
f^{(i)}(0)=\sum_{j=0}^{i}(i-1)(i-2) \cdots(i-j+1) a_{j} h^{(i-j)}(0) \quad(0 \leq i \leq \ell-1)
$$

for which the associated lower triangular Toeplitz matrix

$$
T\left(a_{0}, \ldots, a_{\ell-1}\right)=\left[\begin{array}{cccc}
a_{0} & & \cdots & 0 \\
a_{1} & a_{0} & \cdots & 0 \\
\vdots & \vdots & \ddots & \vdots \\
a_{\ell-1} & a_{\ell-2} & \cdots & a_{0}
\end{array}\right]
$$

has $\left\|T\left(a_{0}, \ldots, a_{\ell-1}\right)\right\| \leq 1$.

Proof. By the characterization (II), $T_{\phi}$ is hyponormal if and only if there exists a function $K$ in $H^{\infty}$ with $\|K\|_{\infty} \leq 1$ and a function $g$ in $H^{\infty}$ such that $\phi=K \bar{\phi}+g$. Hence $T_{\phi}$ is hyponormal if and only if $2 \ell \leq t+s$ by (1) of Corollary 5 in 4 and there exists a function $K$ in $H^{\infty}$ with $\|K\|_{\infty} \leq 1$ and a function $g$ in $H^{\infty}$ such that

$$
\bar{z}^{\ell} \prod_{j=1}^{t}\left(z-\alpha_{j}\right) \prod_{j=1}^{s}\left(z-\beta_{j}\right)=K z^{\ell} \prod_{j=1}^{t}\left(\bar{z}-\bar{\alpha}_{j}\right) \prod_{j=1}^{s}\left(\bar{z}-\bar{\beta}_{j}\right)+g .
$$

The above equality can be written as follows:

$$
f=K z^{2 \ell-(t+s)} h+z^{\ell} G,
$$

where $G=g / \prod_{j=1}^{t}\left(1-\bar{\alpha}_{j} z\right) \prod_{j=1}^{s}\left(1-\bar{\beta}_{j} z\right)$. Since $z^{(t+s)-2 \ell}\left(f-z^{\ell} G\right)=K h$,

$$
z^{(t+s)-2 \ell}\left(f-z^{\ell} G\right) \prod_{j=1}^{s}\left(z-\beta_{j}\right)=K \prod_{j=1}^{s}\left(1-\bar{\beta}_{j} z\right) .
$$

This implies that $K$ is divisible in $H^{\infty}$ by $z^{(t+s)-2 \ell}$ because $\left|\beta_{j}\right| \leq 1$. Hence, if $k=z^{2 \ell-(t+s)} K$, then $k$ belongs to $H^{\infty}$ and $f=k h+z^{\ell} G$. Hence $T_{\phi}$ is hyponormal if and only if $2 \ell \leq t+s$ and there exists a function $k \in H^{\infty}$ with $\|k\|_{\infty} \leq 1$ such that

$$
f^{(i)}(0)=\sum_{j=0}^{i}{ }_{i} C_{j} k^{(j)}(0) h^{(i-j)}(0) \quad(0 \leq i \leq \ell-1),
$$


where ${ }_{i} C_{j}=i ! / j !(i-j) !$. Put $k=\sum_{j=0}^{\infty} a_{j} z^{j}$. Then $k^{(j)}(0)=j ! a_{j}$ and so

$$
f^{(i)}(0)=\sum_{j=0}^{i}(i-1)(i-2) \cdots(i-j+1) a_{j} h^{(i-j)}(0)
$$

for $0 \leq i \leq \ell-1$. Now the theorem follows from the Carathéodory-Schur interpolation theorem (cf. [2]).

In the characterization (II) of hyponormality, put $\mathcal{E}(\phi)=\left\{k \in H^{\infty} ; \phi=\right.$ $k \bar{\phi}+g, g \in H^{\infty}$, and $\left.\|k\|_{\infty} \leq 1\right\} \cdot \mathcal{E}(\phi)$ has been studied and it may contain more than two elements (see [5]). Hence the $k$ in the proof of Theorem 1 may not be unique in general, and so $\left(a_{j}\right)_{j=0}^{\ell}$ may not be unique.

By a result in the previous paper [5. Corollary 5], if $\left\{1 / \bar{\beta}_{j}\right\}_{j=1}^{s} \subseteq\left\{\alpha_{j}\right\}_{j=1}^{t}$ (see Theorem (1), then $T_{\phi}$ is hyponormal. Here we give a necessary and sufficient condition for hyponormality of $T_{\phi}$ in terms of a relation between $\left\{\alpha_{j}\right\}_{j=1}^{t}$ and $\left\{\beta_{j}\right\}_{j=1}^{s}$ when $\ell=1$ or 2 .

Corollary 1. Let $\ell=1$ in Theorem 1. Then $T_{\phi}$ is hyponormal if and only if

$$
\prod_{j=1}^{t}\left|\alpha_{j}\right| \times \prod_{j=1}^{s}\left|\beta_{j}\right| \leq 1 .
$$

When $t=0$ or $s=0$, we assume

$$
\prod_{j=1}^{t}\left|\alpha_{j}\right|=1 \quad \text { or } \quad \prod_{j=1}^{s}\left|\beta_{j}\right|=1 .
$$

Proof. By Theorem 1 $T_{\phi}$ is hyponormal if and only if $f(0)=a_{0} h(0)$ and $\left|a_{0}\right| \leq$ 1.

Corollary 2. Let $\ell=2$ in Theorem 1, Then $T_{\phi}$ is hyponormal if and only if there exist constants $a_{0}, a_{1}$ such that $\left|a_{1}\right| \leq 1-\left|a_{0}\right|^{2}$ and

$$
\begin{gathered}
\prod_{j=1}^{t}\left|\alpha_{j}\right| \times \prod_{j=1}^{s}\left|\beta_{j}\right| \leq 1 \\
\sum_{k=1}^{t}\left\{\left(1-\left|\alpha_{k}\right|^{2}\right) \prod_{j \neq k}^{t}\left(-\alpha_{j}\right)\right\}=a_{0} \sum_{k=1}^{s} \frac{\left(\left|\beta_{k}\right|^{2}-1\right)}{\beta_{k}^{2}} \prod_{j \neq k}^{s}\left(-\frac{1}{\beta_{j}}\right)+a_{1} \prod_{j=1}^{s}\left(-\frac{1}{\beta_{j}}\right) .
\end{gathered}
$$

If $s=0$, then

$$
\sum_{k=1}^{t}\left\{\left(1-\left|\alpha_{k}\right|^{2}\right) \prod_{j \neq k}^{t}\left(-\alpha_{j}\right)\right\}=1
$$

and if $t=0$, then there exist constants $a_{0}, a_{1}$ such that $\left|a_{1}\right| \leq 1-\left|a_{0}\right|^{2}$ and

$$
a_{0} \sum_{k=1}^{s}\left(\left|\beta_{k}\right|^{2}-1\right) \prod_{j \neq k}^{s}\left(-\frac{1}{\beta_{j}}\right)+a_{1} \prod_{j=1}^{s}\left(-\frac{1}{\beta_{j}}\right)=0 .
$$

Proof. By Theorem 1 $T_{\phi}$ is hyponormal if and only if $f(0)=a_{0} h(0), f^{\prime}(0)=$ $a_{0} h(0)+a_{1} h^{\prime}(0)$, and $\left|a_{1}\right| \leq 1-\left|a_{0}\right|^{2}$.

Corollary 3. Let $s=0$ in Theorem 1. Then $T_{\phi}$ is hyponormal if and only if $f^{(i)}(0)=a_{i}(0 \leq i \leq \ell-1)$ and $\left\|T\left(a_{0}, a_{1}, \ldots, a_{l-1}\right)\right\| \leq 1$. 
Proof. By Theorem 1 $T_{\phi}$ is hyponormal if and only if $f^{(i)}(0)=a_{0}$ for $i=0,1,2$ and $\left\|T\left(a_{0}, 0,0\right)\right\|=\left|a_{0}\right| \leq 1$.

Corollary 4. Let $t=0$ in Theorem 1. Then $T_{\phi}$ is hyponormal if and only if $1=a_{0} h(0), \sum_{j=0}^{i} a_{j} h^{(i-j)}(0)=0(1 \leq i \leq \ell-1)$ and $\| T\left(a_{0}, a_{1}, \ldots, a_{\ell-1} \| \leq 1\right.$.

Our corollaries are new and different from Examples 6 and 7 in [6. The author of [6] proved them under some condition $a_{2} \neq 0$ in Example 6. Of course, his result is not for zeros of a polynomial.

\section{ACKNOWLEDGMENT}

The author would like to thank the referee for many comments which improved the original manuscript.

\section{REFERENCES}

[1] C. C. Cowen, Hyponormality of Toeplitz operators, Proc. Amer. Math. Soc. 103(1988), 809812. MR0947663 (89f:47038)

[2] J. A. Ball, I. Gohberg and L. Rodman, Interpolation of Rational Matrix Functions, Operator Theory: Advances and Applications, 45, Birkhäuser-Verlag, Basel, 1990. MR1083145 (92m:47024)

[3] I. S. Hwang and W. Y. Lee, Hyponormality of trigonometric Toeplitz operators, Trans. Amer. Math. Soc. 354(2002), 2461-2474. MR1888332 (2003a:47057)

[4] T. Nakazi and K. Takahashi, Hyponormal Toeplitz operators and extremal problems of Hardy spaces, Trans. Amer. Math. Soc. 338(1993), 753-767. MR1162103 (93j:47040)

[5] I. Schur, Über Potenzreihen die im Innern des Einheitskreises beschränkt sind, J. Reine Angew. Math. 147(1917), 205-232.

[6] K. Zhu, Hyponormal Toeplitz operators with polynomial symbols, Integr. Equat. Oper. Th. 21(1995), 376-381. MR1316550 (95m:47044)

Department of Mathematics, Faculty of Science, Hokkaido University, Sapporo 0600810, JAPAN 